\title{
Physiological Performance of Crossbred Cattle Calves (Karan Fries) under Different Housing Conditions during Different Seasons
}

\author{
Vaibhav N. Sanap ${ }^{*}$, Ashutosh Ludri², Nazir Ahmad Mir, \\ Bharath Kumar ${ }^{4}$ and Kamlesh Kumar Mittal ${ }^{5}$ \\ ${ }^{1}$ Indian Institute of Technology Kanpur, Uttar Pradesh, India \\ ${ }^{2}$ National Dairy Research Institute, Karnal, Haryana, India \\ ${ }^{3}$ Krishi Vigyan Kendra Leh-Ladakh, Jammu \& Kashmir, India \\ ${ }^{4}$ Department of Animal Husbandry and Veterinary Services, Karnataka, India \\ ${ }^{5}$ Department of Animal Husbandry, Uttar Pradesh, India \\ *Corresponding author
}

\begin{tabular}{|l|}
\hline Ke y w o r d s \\
Housing, Crossbred, \\
Calves, \\
Hematology, \\
Hormone
\end{tabular}

\section{Introduction}

The success of dairy industry depends on appropriate calf management. The dairy (1)
Present study was done to examine the seasonal effects of different housing conditions on the performance of crossbred cattle calves (Karan Fries). Thirty healthy female calves of age between 5 and 6 months were selected and divided into five groups (six animals per group) and reared under different housing conditions for a period of 7 months. First group was reared under the scientifically designed calf shade at Livestock Research Centre at National Dairy Research Institute, Karnal (control) and the remaining four groups were reared under different housing conditions identified based on their microenvironment from the nearby village Arinpura, District Karnal. Those were as follows Test Group I - the housing with walls and roof made up of brick and mortar, Test Group II - the housing with walls and roof made up of hatch and mud, Test Group III the housing with brick walls and asbestos roofing, Test Group IV - was simply reared under the tree. The influence of the housing microenvironment on the growth and various other physiological, haematological and endocrinal parameters was studied. The parameters like respiratory rate (RR), skin temperature (ST), white blood count (WBC), red blood cell count (RBC), haemoglobin $(\mathrm{Hb})$, packed cell volume $(\mathrm{PCV})$, growth hormone $(\mathrm{GH})$, cortisol, triiodothyronine $\left(\mathrm{T}_{3}\right)$ and thyroxin $\left(\mathrm{T}_{4}\right)$ were found to be significantly affected by the housing condition provided. Though the feed intake and average daily weight gain of experimental calves was found to be increased with advance of age among all the housing systems, the feed intake and avg. daily weight gain among the calves under different housing systems was found to be significantly affected by seasonal variations. No much variation observed in the pulse rate (PR) and rectal temperature (RT) among the groups. enterprise depends on successful raising of
calves and heifers for replacement of old and
unproductive cattle. Protection from extreme
environmental conditions and proper nutrition 
are the crucial factor in the management of calves for normal growth and development. In India and South East Asian countries, calf rearing practices are not much standardised particularly under rural areas and the proper housing and management is provided to the producing animals only. Calf mortality in rural areas is very due to poor management and shelter facilities (Duguma et al., 2012).

The extreme changes in environmental temperature results in significant loss to the livestock industry, throughout the year. Temperatures above $30^{\circ} \mathrm{C}$ adversely affect the growth and daily weight gain of dairy cattle (Nardone et al., 2006). Under tropical conditions, growth rate is relatively more in spring and autumn seasons than in summer as the temperature load was lower during the former. Heat stress has been linked to decrease in dry matter intake (Blackshaw and Blackshaw, 1994), average daily gain, feed: gain ratio (Silanikove et al., 1987) and the concentrations of hormones associated with growth such as $\mathrm{GH}$ and thyroid hormones (Perera et al., 1985; Ingole, 2012). Exposure to high environmental temperatures leads to an acute increase in plasma cortisol levels (Habeeb et al., 1992) and significant changes in haematological parameters (Coban and Sabuncuoglu, 2005; Broucek et al., 2009).

Coleman et al., (1996) observed a better feed efficiency among the calves housed in shaded plastic hutches than the calves reared in hutches without shade. Spain and Spiers, (1996) carried out a similar experiment and observed a significant difference in the RT, ST and RR between the calves reared in shaded hutches and the normal ones. Razaaque et al., (2009) reported that the average daily live weight gain was significantly higher in calves housed in hutches than conventional housing system (413 versus $113 \mathrm{~g} / \mathrm{h} / \mathrm{d} ; \mathrm{P} \leq 0.0001$ ). Kurtz (1961), comparing the rearing of calves in the building and outside it i.e. in individual hutches, revealed that the calves kept outdoor had better body weight gains and lower mortality as compared to the animals kept in the buildings. Hill et al., (2007) indicated that the conventional housing had a negative impact on growth rates of female calves. Heinrichs et al., (2005) observed a better growth rate in calves housed in open environment hutches than the calves housed in confined crates and concluded that the difference observed may be due to the behavioural satisfaction of calves in open environment hutches. A comfortable resting posture and display of increased social behaviour were earlier observed to be positively associated with an increased growth rate of calves (Andrighetto et al., 1999; Chua et al., 2002).

In rural India, rearing livestock is a secondary occupation employed by farmers and mostly minimal investments are allocated to their housing systems. Usually the animals are reared under tree or an affordable housing system such as Asbestos shed, thatch and brick house is usually entertained. Comparison of haematological, physiological and endocrinological parameters and growth rate of calves reared in the housing systems mentioned above with that of the conventional system could indicate an ideal affordable housing system best suitable for Indian tropical environmental conditions.

\section{Materials and Methods}

The study was conducted at Livestock Research Centre (LRC) of National Dairy Research Institute (NDRI), Karnal and Village Arainpura, District Karnal. Thirty healthy female Crossbred (CB) calves, aged between 5 and 6 months were selected from the LRC, NDRI and village Arainpura. The animals were divided into 5 groups $(n=6)$ and were reared under following housing conditions same plane of nutrition. Control Group 1:- the 
scientifically designed calf shade at Livestock Research Centre at N.D.R.I. Karnal, Test Group I - the housing with walls and roof made up of brick and mortar, Test Group II the housing with walls and roof made up of hatch and mud, Test Group III - the housing with brick walls and asbestos roofing and under tree without any Test Group IV - was simply reared under the tree. All the experimental animals prior to start of actual experimental work were kept in a shade for adaptation in experimental conditions for 15 days to avoid variations in data recordings due to handling stress of these animals.

\section{Feed intake}

Daily DM intake was recorded from the feed offered and residue left on each day throughout the experiment period. The DM of different feed ingredients was recorded once every week.

\section{Body weight}

Body weight of the calves was measured on weekly basis with the help of electronic weighing balance.

\section{Physiological reactions}

The entire physiological variables were recorded at morning $(7 \mathrm{am})$ and afternoon (3 $\mathrm{pm})$. RR of each animal was recorded from visual observation of inward and outward movement of the flank. One outward and inward movement was counted as one respiration and the $R R$ are expressed in breaths per minute. PR of the animals was counted by feeling the pulsation of middle coccygeal artery at the base of the tail and the results are expressed in beats per minute. RT ${ }^{\circ} \mathrm{C}$ was recorded using digital thermometer by keeping the thermometer in contact with the rectal mucosa for about 2 minutes. The peripheral ST at different anatomical sites of the experimental animals viz., forehead, dorsal, ventral and flank regions were recorded using Non-contact Telethermometer (Raytek, Model Raynger ST2L, M/s. Surrey Scientific, Surrey, U.K.) by keeping it 2-3 inches away from the surface of the desired site.

\section{Haematology and endocrine profile}

Whole blood specimens from experimental animals were obtained by acceptable veterinary techniques using EDTA an anticoagulant in a vacutainer. An aliquot of blood was taken immediately and analysed for blood parameters viz. WBC, $\mathrm{RBC}, \mathrm{Hb}$ concentration, haematocrit, by using BC-2800 Vet blood analyser. Plasma was separated and stored at $-20^{\circ} \mathrm{C}$ until analyzed for the $\mathrm{GH}, \mathrm{T}_{4}$, and $\mathrm{T}_{3}$ concentrations. GH (catalogue No. SEA044Bo) and Cortisol (catalogue no. CEA462Bo) where determined in plasma sample of calves by using the ELIZA kit of the company Uscn Life Sciences Inc. Export Processing Zone Building F, Wuhan, Hubei 430056, PRC. $\mathrm{T}_{3}$ and $\mathrm{T}_{4}$ where determined in plasma samples by using "Bovine $\mathrm{T}_{3}$ RIA Kit" (catalogue No. 3288) and "Bovine $\mathrm{T}_{4}$ RIA Kit" (catalogue No. 3289) respectively supplied by Beckman Coulter Chemical Company.

\section{Statistical analysis}

The statistical analysis was carried out to find the mean \pm SE (Snedecor and Cochran, 1994). Two way analysis of variance (ANOVA) was done to find out the significant difference between housing groups.

\section{Results and Discussion}

\section{Physiological parameters}

It can be noted all the test groups from village conditions exhibited higher readings for RR 
and PR during hot humid season followed by during the hot dry season (Table 1) whereas the control group from LRC, NDRI, Karnal exhibited the normal RR and PR during all the seasons. Among the field housing systems, animals housed under brick roof had significantly $(p<0.05)$ lower $R R$ and PR, whereas significantly $(\mathrm{p}<0.05)$ higher rates was observed among animals housed under test group 2. Significant $(\mathrm{p}<0.05)$ differences among the housing systems during morning and afternoon was noted.

A significant difference $(\mathrm{P}<0.01)$ among different experimental groups was observed for ST, whereas the RT was not significantly affected. Among all the test groups the lowest of ST $\left(36.86^{\circ} \mathrm{C}\right)$ was recorded under control group. The perusal of data for each group revealed that there was an oscillation of 1.11 to $2.05^{\circ} \mathrm{C}$ in $\mathrm{RT}$ during 24 hours. The results of present study are in agreement with the findings of O'Brien et al.,(2010) who also reported that at 4:00 $\mathrm{PM}$, heat stressed calves had a $1.15^{\circ} \mathrm{C}$ increase in $\mathrm{RT}$, and $>2.5$-fold increase in $\mathrm{RR}$.

Guyton (1986) also suggested that the rise in body temperature is relatively more at high ambient temperature with high humidity. The physiological reactions and ST during hot dry and hot humid season were significantly different from the spring season but there were no significant effect on RT and PR but RR increased significantly which may suggest the ability of calves to dissipate heat through increased RR under all type of housing systems.

Similar findings were observed by Prasanpanich et al., (2002) who reported that HF crossbred cows housed outdoor showed higher RT, RR and ST. Dandage (2009) also reported positive correlation between RT, humidity and other physiological responses in the Karan Fries (crossbred) cattle as reported in the present study.

\section{Hematological parameters}

The RBC count, WBC count, $\mathrm{Hb} \%$ and PCV was found to be significantly affected $(\mathrm{P}<0.01)$ by housing conditions (Table 2$)$. The mean $\mathrm{RBC}$ count in control group ranged between 7.23 and $8.18 \times 10^{12} / 1$ with the highest value observed during hot humid season. Among field housing systems, highest RBC counts were observed Test Group IV, Test Group II and Test Group III especially during hot humid season.

During hot humid season RBC count was reasonably higher under almost all the experimental groups. In control group the highest WBC count was recorded during spring season, whereas under field conditions a marked variation in WBC count between different housing systems and different seasons was observed. The highest values of WBC count under field condition were reported under Test Group IV followed by Test Group III and Test Group II particularly during dry hot season.

The increase in $\mathrm{Hb}$ concentrations during dry hot and hot humid seasons may be due to haemoconcentration in hot environments and hemodilution during spring season which may be considered as thermo neutral condition. Hemoglobin concentration increases during heat stress due to hemoconcentration (Shebaita and Kamal, 1973; Marai et al., 1995). The PCV were significantly higher during summer than spring in calves under all field housing conditions.

In contrary Marai et al., (1997) reported decreased PCV during summer due to red blood cell destruction and/or to hemodilution. Singh (1983) have reported a significant depression in PCV for the heat stressed cattle and they attributed that to the hemodilution effect where more water is transported in the circulatory system for evaporative cooling. 
Table.1 Mean \pm S.E.M values for physiological variables under different housing conditions and seasons

\begin{tabular}{|c|c|c|c|c|c|c|c|c|c|c|c|c|}
\hline \multirow[t]{3}{*}{ Season } & \multirow{3}{*}{$\begin{array}{c}\text { Age } \\
\text { (mon } \\
\text { ths) }\end{array}$} & \multirow{3}{*}{$\begin{array}{l}\text { Par } \\
\text { ame } \\
\text { ters }\end{array}$} & \multirow{2}{*}{\multicolumn{2}{|c|}{ Control Group }} & \multicolumn{8}{|c|}{ Field } \\
\hline & & & & & \multicolumn{2}{|c|}{ Test Group I } & \multicolumn{2}{|c|}{ Test Group II } & \multicolumn{2}{|c|}{ Test Group III } & \multicolumn{2}{|c|}{ Test Group IV } \\
\hline & & & $\mathbf{M}$ & $\mathbf{E}$ & $\mathbf{M}$ & $\mathbf{E}$ & $\mathbf{M}$ & $\mathbf{E}$ & $\mathbf{M}$ & $\mathbf{E}$ & $\mathbf{M}$ & $\mathbf{E}$ \\
\hline \multirow[t]{8}{*}{ Spring } & \multirow[t]{4}{*}{6} & $\mathrm{RR}$ & $21.00^{\mathbf{a x}} \pm 1.10$ & $30.33^{\mathrm{bx}} \pm 1.73$ & $21.17^{\mathbf{a x}} \pm 1.25$ & $30.67^{b x} \pm 2.75$ & $20.50^{\mathrm{ax}} \pm 1.57$ & $39.33^{\mathrm{cx}} \pm 2.54$ & $22.33^{\mathrm{ax}} \pm 1.12$ & $32.00^{b x} \pm 3.08$ & $19.50^{\mathrm{dx}} \pm 1.59$ & $37.17^{\mathrm{cx}} \pm 4.23$ \\
\hline & & PR & $83.67^{\mathrm{ax}} \pm 3.11$ & $94.17^{b \mathbf{b x}} \pm 2.74$ & $84.33^{a x} \pm 2.54$ & $93.00^{\mathrm{bx}} \pm 2.56$ & $86.33^{\mathrm{ax}} \pm 2.64$ & $95.33^{b x} \pm 2.22$ & $84.83^{\mathrm{ax}} \pm 3.87$ & $93.17^{b x} \pm 1.76$ & $90.50^{\mathbf{a x}} \pm 3.36$ & $95.00^{\mathbf{b x}} \pm 0.68$ \\
\hline & & RT & $38.84^{\mathbf{a x}} \pm 0.19$ & $38.53^{b x} \pm 0.14$ & $38.79^{a x} \pm 0.15$ & $38.94^{\mathbf{a x}} \pm 0.28$ & $38.44^{\mathbf{b x}} \pm 0.12$ & $39.06^{\mathbf{c x}} \pm 0.24$ & $38.93^{\mathbf{a x}} \pm 0.19$ & $38.98^{\mathbf{a x}} \pm 0.36$ & $38.56^{\mathbf{a x}} \pm 0.24$ & $39.09^{\mathrm{cy}} \pm 0.17$ \\
\hline & & ST & $31.61^{\mathrm{ax}} \pm 0.38$ & $34.39^{b x} \pm 0.44$ & $31.63^{a x} \pm 0.43$ & $32.44^{\mathrm{ax}} \pm 0.57$ & $32.87^{\mathbf{a x}} \pm 0.71$ & $33.94^{b x} \pm 1.0$ & $32.41^{\mathbf{a x}} \pm 0.53$ & $33.66^{b x} \pm 0.95$ & $32.88^{\mathbf{a x}} \pm 0.58$ & $32.30^{\mathrm{ax}} \pm 0.67$ \\
\hline & \multirow[t]{4}{*}{7} & $\mathrm{RR}$ & $20.17^{\mathbf{a x}} \pm 1.49$ & $36.67^{\text {by }} \pm 1.05$ & $21.83^{\mathrm{ax}} \pm 1.76$ & $37.00^{\text {by }} \pm 2.86$ & $22.67^{a x} \pm 1.58$ & $37.50^{\mathrm{bx}} \pm 3.33$ & $23.67^{b x} \pm 1.33$ & $34.17^{\mathrm{cx}} \pm 1.17$ & $21.33^{\mathrm{ay}} \pm 2.36$ & $31.83^{\mathrm{dy}} \pm 4.96$ \\
\hline & & PR & $90.33^{\text {ay }} \pm 2.72$ & $93.83^{b x} \pm 2.15$ & $87.17^{\mathrm{ay}} \pm 2.86$ & $96.00^{\text {by }} \pm 1.71$ & $87.50^{\mathrm{ax}} \pm 3.52$ & $93.17^{b x} \pm 2.86$ & $86.50^{\mathrm{ay}} \pm 2.99$ & $95.50^{\text {by }} \pm 1.52$ & $89.00^{\mathbf{a x}} \pm 3.94$ & $95.17^{\mathbf{b x}} \pm 0.75$ \\
\hline & & RT & $38.41^{\mathrm{ax}} \pm 0.21$ & $38.33^{\mathbf{a x}} \pm 0.44$ & $38.54^{\mathrm{ax}} \pm 0.17$ & $39.06^{\mathbf{b x}} \pm 0.30$ & $38.53^{\mathrm{ax}} \pm 0.12$ & $39.23^{\text {by }} \pm 0.41$ & $38.68^{\mathbf{a x}} \pm 0.17$ & $39.13^{\mathbf{b x}} \pm 0.28$ & $38.34^{\mathbf{a x}} \pm 0.10$ & $38.65^{\mathbf{b x}} \pm 0.24$ \\
\hline & & ST & $32.27^{\mathbf{a x}} \pm 0.37$ & $35.77^{\mathbf{c x}} \pm 0.34$ & $33.20^{\mathbf{b y}} \pm 0.45$ & $34.48^{\mathrm{cy}} \pm 0.75$ & $32.33^{\mathbf{a x}} \pm 0.47$ & $35.05^{\mathrm{cy}} \pm 1.05$ & $33.30^{\mathbf{b x}} \pm 0.20$ & $34.93^{c x} \pm 0.64$ & $33.89^{b x} \pm 1.08$ & $35.01^{\mathrm{cy}} \pm 0.52$ \\
\hline \multirow[t]{8}{*}{ Dry Hot } & \multirow[t]{4}{*}{8} & $\mathrm{RR}$ & $15.83^{\mathrm{ay}} \pm 1.11$ & $41.00^{\mathrm{bz}} \pm 1.63$ & $20.83^{\mathrm{cx}} \pm 1.94$ & $43.67^{\text {by }} \pm 0.92$ & $22.00^{\mathrm{cx}} \pm 0.73$ & $63.00^{\mathrm{dz}} \pm 2.66$ & $22.83^{\mathrm{cx}} \pm 1.30$ & $57.33^{\mathrm{dy}} \pm 4.20$ & $18.17^{\mathrm{ax}} \pm 2.34$ & $52.67^{\mathrm{dz}} \pm 5.11$ \\
\hline & & PR & $87.00^{\mathbf{a x}} \pm 2.91$ & $94.00^{\mathbf{b x}} \pm 2.68$ & $84.50^{\mathbf{a x}} \pm 2.31$ & $93.67^{b x} \pm 3.06$ & $84.50^{\mathrm{ay}} \pm 2.54$ & $91.50^{\text {by }} \pm 1.91$ & $83.50^{\mathbf{a x}} \pm 1.23$ & $92.33^{b x} \pm 1.31$ & $84.00^{\mathbf{a y}} \pm 2.14$ & $94.83^{\mathrm{cx}} \pm 1.49$ \\
\hline & & RT & $38.30^{\mathbf{a x}} \pm 0.20$ & $39.33^{\text {by }} \pm 0.19$ & $38.62^{a x} \pm 0.17$ & $38.57^{\mathbf{a x}} \pm 0.39$ & $38.53^{\mathbf{a x}} \pm 0.16$ & $39.27^{\text {by }} \pm 0.28$ & $38.76^{\mathbf{a x}} \pm 0.18$ & $39.26^{\mathbf{b x}} \pm 0.37$ & $38.30^{\mathbf{a x}} \pm 0.16$ & $39.90^{\mathrm{bz}} \pm 0.23$ \\
\hline & & ST & $33.37^{\mathbf{a x}} \pm 0.84$ & $36.91^{\mathbf{c x}} \pm 0.26$ & $34.31^{\mathbf{b y}} \pm 0.88$ & $36.34^{\mathrm{cz}} \pm 0.24$ & $32.77^{\mathbf{a x}} \pm 0.69$ & $37.33^{\mathrm{cz}} \pm 1.17$ & $33.50^{\mathbf{a x}} \pm 0.92$ & $38.48^{\mathrm{dy}} \pm 0.98$ & $35.05^{\text {by }} \pm 0.70$ & $39.51^{\mathrm{dz}} \pm 0.70$ \\
\hline & \multirow[t]{4}{*}{9} & $\mathrm{RR}$ & $19.17^{\mathrm{ax}} \pm 1.92$ & $43.33^{\mathrm{cz}} \pm 2.20$ & $18.83^{\mathrm{ay}} \pm 1.58$ & $45.67^{\mathrm{cy}} \pm 1.96$ & $21.00^{\mathrm{ax}} \pm 1.69$ & $67.67^{\mathrm{dz}} \pm 5.40$ & $22.00^{\mathbf{a x}} \pm 0.97$ & $69.33^{\mathrm{dz}} \pm 2.56$ & $16.83^{\mathrm{bx}} \pm 0.87$ & $63.50^{\mathrm{dv}} \pm 6.05$ \\
\hline & & PR & $89.67^{\text {ay }} \pm 3.31$ & $93.67^{b x} \pm 1.74$ & $87.67^{\mathrm{ay}} \pm 3.55$ & $94.17^{\text {by }} \pm 1.74$ & $89.17^{\mathrm{az}} \pm 3.21$ & $91.83^{\text {by }} \pm 2.23$ & $88.50^{\mathrm{az}} \pm 2.81$ & $92.83^{b x} \pm 1.51$ & $86.83^{\mathrm{ay}} \pm 3.54$ & $90.67^{\text {by }} \pm 1.09$ \\
\hline & & RT & $38.52^{\mathbf{a x}} \pm 0.16$ & $38.73^{\mathbf{a x}} \pm 0.27$ & $38.38^{\mathbf{a x}} \pm 0.36$ & $38.95^{\mathbf{b x}} \pm 0.35$ & $38.55^{\mathbf{a x}} \pm 0.09$ & $38.89^{\mathbf{b x}} \pm 0.25$ & $38.85^{\mathbf{a x}} \pm 0.21$ & $38.77^{\mathbf{b x}} \pm 0.25$ & $38.58^{\mathbf{a x}} \pm 0.15$ & $39.23^{\mathrm{cy}} \pm 0.24$ \\
\hline & & ST & $34.16^{\text {ay }} \pm 0.78$ & $37.76^{\mathrm{cy}} \pm 0.18$ & $35.23^{b z} \pm 0.59$ & $36.15^{\mathrm{bz}} \pm 0.42$ & $33.38^{\mathbf{a x}} \pm 0.46$ & $39.68^{\mathbf{c z}} \pm 1.57$ & $33.93^{\mathbf{a x}} \pm 0.43$ & $38.60^{\mathrm{cy}} \pm 1.18$ & $34.52^{\mathrm{ax}} \pm 0.52$ & $41.73^{\mathrm{dv}} \pm 2.03$ \\
\hline \multirow{12}{*}{$\begin{array}{c}\text { Hot } \\
\text { Humid }\end{array}$} & \multirow[t]{4}{*}{10} & $\mathrm{RR}$ & $21.00^{\mathrm{ax}} \pm 1.03$ & $46.50^{\mathrm{bz}} \pm 2.62$ & $19.33^{\mathrm{ay}} \pm 1.45$ & $46.67^{\text {by }} \pm 1.65$ & $19.33^{a x} \pm 0.92$ & $67.67^{\mathrm{cz}} \pm 2.20$ & $23.67^{\mathrm{bx}} \pm 1.48$ & $67.50^{\mathrm{cz}} \pm 1.59$ & $23.83^{\text {by }} \pm 0.40$ & $57.00^{\mathrm{dz}} \pm 5.93$ \\
\hline & & PR & $88.67^{\mathrm{ax}} \pm 3.52$ & $95.33^{\mathbf{b x}} \pm 0.80$ & $88.83^{\mathrm{ay}} \pm 2.86$ & $91.67^{\mathrm{bz}} \pm 2.80$ & $85.00^{\mathbf{a x}} \pm 2.78$ & $89.33^{\mathrm{az}} \pm 2.62$ & $87.67^{\mathrm{az}} \pm 2.73$ & $94.83^{\text {by }} \pm 0.60$ & $89.50^{\mathrm{ax}} \pm 3.61$ & $93.83^{b x} \pm 1.54$ \\
\hline & & $\mathrm{RT}$ & $38.34^{\mathbf{a x}} \pm 0.19$ & $39.14^{\text {by }} \pm 0.27$ & $38.75^{b \mathbf{b}} \pm 0.16$ & $39.03^{\mathbf{b x}} \pm 0.34$ & $38.83^{\text {by }} \pm 0.20$ & $39.49^{\mathrm{cy}} \pm 0.15$ & $38.75^{b x} \pm 0.18$ & $39.06^{\mathbf{b x}} \pm 0.27$ & $38.74^{b x} \pm 0.15$ & $39.39^{\mathrm{cy}} \pm 0.19$ \\
\hline & & ST & $34.37^{\mathrm{ax}} \pm 0.46$ & $36.31^{b x} \pm 0.29$ & $33.14^{\mathrm{ay}} \pm 0.49$ & $36.86^{\mathrm{bz}} \pm 0.46$ & $33.48^{\mathbf{a x}} \pm 0.78$ & $38.69^{\mathrm{bz}} \pm 1.27$ & $32.88^{\mathbf{a x}} \pm 0.71$ & $39.24^{\mathrm{cy}} \pm 1.24$ & $33.69^{a x} \pm 1.18$ & $40.39^{\mathrm{cv}} \pm 1.71$ \\
\hline & \multirow[t]{4}{*}{11} & $\mathrm{RR}$ & $20.00^{\mathrm{ax}} \pm 1.59$ & $56.67^{\mathrm{bv}} \pm 3.88$ & $22.00^{\mathbf{a x}} \pm 0.93$ & $55.67^{\mathrm{bz}} \pm 3.40$ & $20.00^{\mathrm{ax}} \pm 1.26$ & $64.33^{\mathrm{cz}} \pm 3.44$ & $25.17^{\text {by }} \pm 1.62$ & $71.50^{\mathrm{dv}} \pm 2.85$ & $22.67^{\text {ay }} \pm 0.80$ & $57.67^{\mathrm{bz}} \pm 6.28$ \\
\hline & & PR & $87.67^{\mathrm{ax}} \pm 3.81$ & $92.17^{\mathbf{b y}} \pm 2.36$ & $83.33^{a x} \pm 2.14$ & $92.83^{b x} \pm 2.51$ & $86.17^{\mathbf{a x}} \pm 2.75$ & $90.17^{\mathrm{bz}} \pm 1.89$ & $85.00^{\mathrm{ax}} \pm 1.98$ & $91.50^{\mathrm{bz}} \pm 1.06$ & $87.50^{\mathrm{ax}} \pm 2.77$ & $95.50^{\mathbf{b x}} \pm 1.65$ \\
\hline & & RT & $38.23^{\mathbf{a x}} \pm 0.11$ & $39.03^{\mathbf{c x}} \pm 0.21$ & $38.28^{\mathrm{ay}} \pm 0.12$ & $39.08^{\mathrm{cy}} \pm 0.44$ & $38.45^{\mathrm{ax}} \pm 0.19$ & $39.25^{\mathrm{cy}} \pm 0.33$ & $38.70^{b x} \pm 0.24$ & $39.00^{\mathbf{c x}} \pm 0.19$ & $38.47^{\mathbf{b x}} \pm 0.17$ & $39.56^{\mathrm{dz}} \pm 0.22$ \\
\hline & & ST & $31.20^{\mathbf{a x}} \pm 0.84$ & $36.75^{b x} \pm 0.17$ & $34.04^{\mathbf{b y}} \pm 0.71$ & $36.72^{\mathrm{bz}} \pm 0.44$ & $34.15^{\text {by }} \pm 1.30$ & $40.90^{\mathrm{cv}} \pm 1.63$ & $34.05^{b x} \pm 0.83$ & $39.79^{\mathrm{cz}} \pm 1.13$ & $35.19^{\text {by }} \pm 0.94$ & $39.99^{\mathrm{cz}} \pm 1.06$ \\
\hline & \multirow[t]{4}{*}{12} & $\mathrm{RR}$ & $18.83^{\text {ay }} \pm 1.33$ & $55.00^{\mathrm{dv}} \pm 3.64$ & $21.67^{b x} \pm 0.80$ & $57.67^{\mathrm{dz}} \pm 4.74$ & $22.50^{\mathrm{bx}} \pm 1.26$ & $73.17^{\mathrm{ev}} \pm 2.15$ & $25.50^{\mathrm{cy}} \pm 1.31$ & $70.50^{\mathrm{ev}} \pm 2.13$ & $20.83^{b x} \pm 1.17$ & $69.50^{\mathrm{ev}} \pm 4.22$ \\
\hline & & PR & $86.00^{\mathrm{ax}} \pm 2.10$ & $93.67^{b \mathbf{b x}} \pm 1.12$ & $85.83^{\mathbf{a x}} \pm 3.07$ & $92.33^{b x} \pm 2.44$ & $86.00^{\mathbf{a x}} \pm 2.21$ & $93.67^{b x} \pm 2.23$ & $86.50^{\mathrm{ay}} \pm 2.80$ & $93.17^{b x} \pm 1.40$ & $86.83^{a x} \pm 3.76$ & $91.83^{\mathrm{by}} \pm 1.42$ \\
\hline & & $\mathrm{RT}$ & $38.65^{\mathrm{ax}} \pm 0.15$ & $39.25^{\mathbf{c y}} \pm 0.42$ & $38.63^{\mathrm{ax}} \pm 0.22$ & $39.11^{\mathbf{c y}} \pm 0.31$ & $38.50^{\mathbf{a x}} \pm 0.08$ & $38.92^{\mathrm{ax}} \pm 0.25$ & $38.52^{\mathbf{a x}} \pm 0.19$ & $38.97^{\mathbf{b x}} \pm 0.24$ & $38.63^{a x} \pm 0.15$ & $39.31^{\mathrm{cy}} \pm 0.18$ \\
\hline & & ST & $34.55^{\mathrm{ay}} \pm 0.78$ & $36.50^{\mathbf{b x}} \pm 0.15$ & $34.87^{\mathrm{az}} \pm 1.20$ & $36.57^{\mathrm{bz}} \pm 0.44$ & $34.78^{\mathrm{ay}} \pm 0.65$ & $37.61^{\mathrm{cz}} \pm 0.70$ & $34.35^{\mathrm{ay}} \pm 0.84$ & $38.51^{\text {cy }} \pm 0.98$ & $34.09^{\mathbf{a x}} \pm 0.60$ & $38.51^{\mathrm{cz}} \pm 0.90$ \\
\hline
\end{tabular}


Table.2 Mean \pm S.E.M values for haematological variable under different housing conditions during different seasons

\begin{tabular}{|c|c|c|c|c|c|c|c|}
\hline \multirow[t]{3}{*}{ Season } & \multirow{3}{*}{$\begin{array}{c}\text { Age } \\
\text { (Months) }\end{array}$} & \multirow[t]{3}{*}{ Parameters } & \multicolumn{5}{|c|}{ Housing Condition } \\
\hline & & & \multirow{2}{*}{$\begin{array}{l}\text { Control } \\
\text { Group }\end{array}$} & \multicolumn{4}{|c|}{ Field } \\
\hline & & & & $\begin{array}{c}\text { Test Group } \\
\text { I }\end{array}$ & $\begin{array}{c}\text { Test Group } \\
\text { II }\end{array}$ & $\begin{array}{c}\text { Test Group } \\
\text { III }\end{array}$ & $\begin{array}{c}\text { Test Group } \\
\text { IV }\end{array}$ \\
\hline \multirow[t]{8}{*}{ Spring } & \multirow[t]{4}{*}{6} & WBC & $11.93^{\mathrm{av}} \pm 0.93$ & $11.63^{\mathbf{a x}} \pm 0.53$ & $10.67^{\mathrm{av}} \pm 2.20$ & $11.73^{\mathrm{av}} \pm 1.08$ & $9.83^{\mathbf{b v}} \pm 0.42$ \\
\hline & & RBC & $7.73^{\mathrm{ax}} \pm 0.61$ & $8.40^{b x} \pm 0.23$ & $8.78^{\mathrm{cx}} \pm 0.28$ & $8.63^{\mathrm{cx}} \pm 0.14$ & $8.85^{\mathrm{cx}} \pm 0.35$ \\
\hline & & $\mathbf{H b}$ & $7.32^{\mathrm{ax}} \pm 0.44$ & $8.67^{\mathbf{b x}} \pm 0.36$ & $8.35^{\mathbf{c x}} \pm 0.37$ & $8.30^{\mathrm{cx}} \pm 1.03$ & $8.37^{\mathbf{c x}} \pm 0.34$ \\
\hline & & PCV & $26.31^{\mathrm{ax}} \pm 1.63$ & $35.17^{\mathbf{b x}} \pm 0.78$ & $31.72^{\mathrm{cy}} \pm 1.97$ & $34.42^{\mathrm{bz}} \pm 1.05$ & $31.50^{\mathrm{cy}} \pm 2.00$ \\
\hline & \multirow[t]{4}{*}{7} & WBC & $11.13^{\mathrm{ax}} \pm 1.67$ & $10.90^{\mathrm{ay}} \pm 1.18$ & $11.57^{\mathrm{bx}} \pm 1.87$ & $12.50^{\mathbf{c x}} \pm 1.76$ & $12.73^{\mathrm{cx}} \pm 0.16$ \\
\hline & & RBC & $7.89^{\mathrm{ax}} \pm 0.63$ & $9.37^{\mathbf{b z}} \pm 0.33$ & $9.50^{\mathrm{bz}} \pm 0.55$ & $9.47^{\mathbf{b y}} \pm 0.57$ & $9.33^{\mathbf{b y}} \pm 0.35$ \\
\hline & & $\mathbf{H b}$ & $7.85^{\mathrm{ay}} \pm 0.33$ & $9.05^{\mathbf{b y}^{\prime}} \pm 0.15$ & $9.30^{\mathbf{c x}} \pm 0.55$ & $8.23^{\mathrm{dx}} \pm 0.28$ & $8.32^{\mathrm{dx}} \pm 0.37$ \\
\hline & & PCV & $28.05^{\mathrm{az}} \pm 1.09$ & $34.78^{\mathrm{by}} \pm 2.46$ & $34.43^{\mathrm{bz}} \pm 0.73$ & $34.52^{\mathrm{bz}} \pm 2.41$ & $36.17^{\mathrm{cz}} \pm 0.97$ \\
\hline \multirow{8}{*}{$\begin{array}{l}\text { Dry } \\
\text { Hot }\end{array}$} & \multirow[t]{4}{*}{8} & WBC & $11.52^{\mathrm{ay}} \pm 1.25$ & $11.10^{\mathbf{b y}} \pm 0.91$ & $11.83^{\mathrm{cx}} \pm 1.57$ & $12.70^{\mathbf{d x}} \pm 1.36$ & $15.73^{\mathrm{ey}} \pm 0.09$ \\
\hline & & RBC & $7.23^{\mathrm{ay}} \pm 0.50$ & $8.72^{b x} \pm 0.37$ & $8.03^{\mathrm{cy}} \pm 0.47$ & $8.45^{b \mathbf{b x}} \pm 0.38$ & $7.83^{\mathrm{dv}} \pm 0.19$ \\
\hline & & Hb & $6.84^{\mathrm{av}} \pm 0.33$ & $8.15^{\mathbf{b z}} \pm 0.30$ & $8.13^{b \mathbf{b x}} \pm 0.15$ & $9.90^{\mathrm{cz}} \pm 0.55$ & $8.65^{\mathrm{dy}} \pm 0.27$ \\
\hline & & PCV & $25.28^{\mathrm{ay}} \pm 1.21$ & $27.83^{\mathbf{b v}} \pm 0.62$ & $28.03^{b \mathbf{b x}} \pm 0.31$ & $29.40^{\mathbf{c y}} \pm 1.12$ & $31.15^{\mathrm{dy}} \pm 1.87$ \\
\hline & \multirow[t]{4}{*}{9} & WBC & $11.28^{\mathbf{a x}} \pm 1.45$ & $9.97^{\mathrm{bz}} \pm 1.64$ & $14.13^{\mathrm{cy}} \pm 1.15$ & $13.00^{\mathrm{dy}} \pm 0.97$ & $13.63^{\mathbf{e u}} \pm 0.94$ \\
\hline & & RBC & $7.34^{\mathrm{av}} \pm 0.36$ & $8.15^{\text {by }} \pm 0.13$ & $8.38^{\mathbf{b y}} \pm 0.33$ & $8.98^{\mathrm{cz}} \pm 0.12$ & $8.42^{\mathrm{bz}} \pm 0.28$ \\
\hline & & Hb & $7.31^{\mathbf{a x}} \pm 0.47$ & $8.93^{b x} \pm 0.17$ & $9.67^{\mathrm{cy}} \pm 0.55$ & $9.40^{\mathbf{c y}} \pm 0.62$ & $8.43^{\mathrm{dx}} \pm 0.41$ \\
\hline & & PCV & $25.96^{\mathbf{a x}} \pm 1.69$ & $32.97^{\text {by }} \pm 1.45$ & $28.02^{\mathrm{cx}} \pm 1.92$ & $34.12^{\mathrm{bz}} \pm 1.71$ & $24.35^{\mathrm{ax}} \pm 1.62$ \\
\hline \multirow{12}{*}{$\begin{array}{c}\text { Hot } \\
\text { Humid }\end{array}$} & \multirow[t]{4}{*}{10} & WBC & $11.43^{\mathrm{ay}} 1.30$ & $10.80^{\text {by }} \pm 1.08$ & $11.55^{\mathrm{cx}} \pm 1.73$ & $12.67^{\mathbf{d x}} \pm 1.41$ & $14.33^{\mathrm{ez}} \pm 1.10$ \\
\hline & & RBC & $7.73^{\mathbf{a x}} \pm 0.31$ & $8.00^{\mathrm{ay}} \pm 0.49$ & $8.18^{\mathrm{by}} \pm 0.58$ & $9.40^{\mathbf{c x}} \pm 0.10$ & $8.43^{\mathrm{dz}} \pm 0.51$ \\
\hline & & $\mathbf{H b}$ & $8.93^{\mathrm{az}} \pm 0.32$ & $8.92^{\mathrm{ax}} \pm 0.19$ & $8.98^{\mathrm{az}} \pm 0.12$ & $9.25^{\mathrm{by}} \pm 0.66$ & $9.10^{\mathrm{bz}} \pm 0.15$ \\
\hline & & PCV & $29.80^{\mathrm{az}} \pm 1.09$ & $32.97^{\text {by }} \pm 0.29$ & $28.78^{\mathrm{ax}} \pm 1.80$ & $32.47^{\mathbf{b v}} \pm 0.86$ & $25.48^{\mathbf{c x}} \pm 1.49$ \\
\hline & \multirow[t]{4}{*}{11} & WBC & $11.32^{\mathbf{a y}} \pm 1.40$ & $10.18^{\mathbf{b z}} \pm 1.48$ & $10.92^{\mathrm{cv}} \pm 2.05$ & $12.60^{\mathbf{d x}} \pm 1.51$ & $14.20^{\mathrm{ez}} \pm 1.17$ \\
\hline & & RBC & $8.18^{\mathrm{az}} \pm 0.77$ & $9.63^{b \mathbf{b z}} \pm 0.49$ & $8.18^{\mathrm{ay}} \pm 0.68$ & $8.85^{\mathrm{cz}} \pm 0.15$ & $9.77^{\mathrm{dy}} \pm 0.33$ \\
\hline & & Hb & $7.63^{\mathrm{ax}} \pm 0.55$ & $8.43^{\mathrm{bz}} \pm 0.38$ & $9.63^{\mathrm{cy}} \pm 0.20$ & $9.55^{\mathrm{cz}} \pm 0.36$ & $9.60^{\mathrm{cz}} \pm 0.29$ \\
\hline & & PCV & $27.51^{\mathrm{az}} \pm 1.98$ & $23.43^{\mathrm{bz}} \pm 0.06$ & $31.08^{\mathrm{cy}} \pm 3.41$ & $26.58^{\mathbf{a x}} \pm 2.01$ & $30.55^{\mathrm{cy}} \pm 3.68$ \\
\hline & \multirow[t]{4}{*}{12} & WBC & $10.72^{\mathrm{az}} \pm 0.58$ & $11.27^{\mathbf{b y}} \pm 0.99$ & $10.23^{\mathrm{az}} \pm 0.67$ & $12.77^{\mathbf{c x}} \pm 1.38$ & $13.33^{\mathrm{dx}} \pm 1.24$ \\
\hline & & RBC & $7.59^{\mathrm{av}} \pm 0.30$ & $9.02^{b z} \pm 0.40$ & $9.33^{\mathrm{cz}} \pm 0.34$ & $9.17^{\mathbf{b y}} \pm 0.04$ & $8.85^{\mathrm{dx}} \pm 0.16$ \\
\hline & & Hb & $8.68^{\mathrm{az}} \pm 0.25$ & $8.87^{a x} \pm 0.16$ & $9.20^{\mathbf{b y}} \pm 0.04$ & $9.12^{\mathbf{b y}} \pm 0.21$ & $9.90^{\mathrm{cz}} \pm 0.59$ \\
\hline & & $\mathbf{V}$ & $25.76^{\alpha} \pm 0.95$ & $21.83^{\mathrm{bz}} \pm 1.81$ & 27. & 99 & $31.72^{\mathrm{cy}} \pm 2.66$ \\
\hline
\end{tabular}

WBC- White Bblood Cells, RBC- Red Blood Bells, Hb-Haemoglobin, PCV-Packed Cell Volume $a$ and $b$ values with different superscript differ significantly $(\mathrm{P}<0.05)$ between columns $x$ and $y$ values with different superscript differ significantly $(P<0.05)$ between rows 
Table.3 Mean \pm S.E.M values for the hormones $(\mathrm{ng} / \mathrm{ml})$ in the plasma at different ages under different housing groups

\begin{tabular}{|c|c|c|c|c|c|c|c|}
\hline \multirow[t]{3}{*}{ Seasons } & \multirow{3}{*}{$\begin{array}{c}\text { Age } \\
\text { (Mont } \\
\text { hs) }\end{array}$} & \multirow{3}{*}{$\begin{array}{c}\text { Para } \\
\text { meters }\end{array}$} & \multicolumn{5}{|c|}{ Housing Condition } \\
\hline & & & \multirow{2}{*}{$\begin{array}{l}\text { Control } \\
\text { Group }\end{array}$} & \multicolumn{4}{|c|}{ Field } \\
\hline & & & & Test Group I & $\begin{array}{c}\text { Test Group } \\
\text { II }\end{array}$ & $\begin{array}{c}\text { Test Group } \\
\text { III }\end{array}$ & $\begin{array}{c}\text { Test Group } \\
\text { IV }\end{array}$ \\
\hline \multirow[t]{8}{*}{ Spring } & \multirow[t]{4}{*}{6} & GH & $4.29^{\mathbf{a x}} \pm 0.59$ & $4.26^{\mathbf{a x}} \pm 0.62$ & $4.28^{\mathbf{a x}} \pm 0.21$ & $4.94^{\mathbf{a x}} \pm 0.32$ & $3.05^{\mathbf{b x}} \pm 0.31$ \\
\hline & & Cortisol & $2.19^{\mathrm{ax}} \pm 0.59$ & $4.32^{\mathbf{b v}} \pm 0.53$ & $3.43^{\mathbf{c v}} \pm 0.16$ & $4.24^{\mathrm{av}} \pm 0.50$ & $2.36^{\mathbf{a v}} \pm 0.27$ \\
\hline & & T3 & $2.17^{\mathbf{a x}} \pm 0.01$ & $1.68^{\mathbf{b x}} \pm 0.06$ & $1.18^{\mathbf{c x}} \pm 0.13$ & $1.98^{\mathrm{ax}} \pm 0.10$ & $0.98^{\mathbf{d x}} \pm 0.02$ \\
\hline & & T4 & $51.52^{a x} \pm 4.60$ & $41.82^{b x} \pm 1.94$ & $35.92^{\mathrm{cx}} \pm 2.74$ & $41.11^{b x} \pm 5.71$ & $24.81^{\mathrm{dx}} \pm 1.90$ \\
\hline & \multirow[t]{4}{*}{7} & GH & $5.62^{\mathrm{ay}} \pm 0.84$ & $7.14^{\mathrm{bz}} \pm 0.56$ & $3.73^{\mathrm{cx}} \pm 0.38$ & $6.81^{\text {by }} \pm 0.50$ & $3.95^{\mathrm{cx}} \pm 0.96$ \\
\hline & & Cortisol & $2.64^{\mathrm{ay}} 0.60$ & $2.94^{\mathrm{ax}} \pm 0.63$ & $3.64^{\mathbf{b v}} \pm 0.15$ & $4.09^{\mathbf{c v}} \pm 0.53$ & $4.64^{d x} \pm 0.56$ \\
\hline & & T3 & $2.12^{\mathrm{ax}} \pm 0.05$ & $2.18^{\mathrm{ay}} \pm 0.01$ & $2.08^{\mathrm{ay}} \pm 0.03$ & $1.36^{\mathbf{b y}} \pm 0.23$ & $1.12^{\text {by }} \pm 0.07$ \\
\hline & & T4 & $46.29^{\mathbf{a x}} \pm 3.32$ & $40.88^{\mathbf{b x}} \pm 3.16$ & $36.52^{\mathrm{cx}} \pm 2.10$ & $48.20^{\mathrm{ay}} \pm 3.00$ & $36.98^{\mathrm{cy}} \pm 0.17$ \\
\hline \multirow{8}{*}{$\begin{array}{l}\text { Dry } \\
\text { Hot }\end{array}$} & \multirow[t]{4}{*}{8} & GH & $12.05^{\mathrm{az}} \pm 2.08$ & $4.10^{b z} \mathrm{x} \pm 0.42$ & $5.35^{\mathrm{cy}} \pm 0.53$ & $4.77^{\mathbf{b x}} \pm 0.47$ & $4.96^{\mathbf{b y}} \pm 0.36$ \\
\hline & & Cortisol & $3.73^{\mathrm{az}} \pm 0.17$ & $3.22^{\mathbf{b y}} \pm 0.10$ & $3.18^{\mathbf{b v}} \pm 0.24$ & $5.69^{\mathrm{cx}} \pm 0.35$ & $5.27^{\mathrm{cy}} \pm 0.36$ \\
\hline & & T3 & $2.00^{\mathrm{ax}} \pm 0.05$ & $1.37^{\mathbf{b x}} \pm 0.05$ & $1.15^{b \mathbf{b x}} \pm 0.06$ & $0.79^{\mathrm{cz}} \pm 0.20$ & $0.84^{\mathrm{cx}} \pm 0.11$ \\
\hline & & T4 & $43.03^{\mathrm{ax}} \pm 3.88$ & $35.85^{\mathrm{by}} \pm 1.25$ & $25.23^{\mathrm{cy}} \pm 2.91$ & $26.95^{\mathrm{cz}} \pm 2.77$ & $22.05^{d x} \pm 2.39$ \\
\hline & \multirow[t]{4}{*}{9} & GH & $11.94^{\mathrm{az}} \pm 1.84$ & $9.97^{\mathbf{d v}} \pm 1.76$ & $4.23^{\mathbf{b x}} \pm 0.46$ & $5.35^{\mathbf{b x}} \pm 0.32$ & $3.93^{\mathrm{cx}} \pm 0.37$ \\
\hline & & Cortisol & $5.41^{\mathbf{a v}} \pm 0.39$ & $4.33^{\mathbf{b v}} \pm 0.02$ & $14.79^{\mathbf{c x}} \pm 0.45$ & $8.64^{\mathrm{dy}} \pm 1.19$ & $12.93^{\mathrm{ez}} \pm 0.61$ \\
\hline & & T3 & $0.99^{\mathrm{ay}^{\mathrm{a}}} \pm 0.06$ & $0.77^{\mathbf{b z}} \pm 0.00$ & $0.45^{\mathrm{cz}} \pm 0.11$ & $0.24^{\mathrm{dv}} \pm 0.10$ & $0.58^{\mathbf{c v}} \pm 0.07$ \\
\hline & & T4 & $39.76^{\mathbf{a y}} \pm 3.06$ & $34.33^{\mathrm{ay}} \pm 4.41$ & $25.14^{\mathbf{b y}} \pm 1.44$ & $37.74^{\mathrm{av}} \pm 4.79$ & $24.35^{\mathbf{b x}} \pm 0.78$ \\
\hline \multirow{12}{*}{$\begin{array}{c}\text { Hot } \\
\text { Humid }\end{array}$} & \multirow[t]{4}{*}{9} & GH & $6.07^{\mathrm{ay}} \pm 1.49$ & $7.56^{\mathrm{az}} \pm 0.54$ & $4.82^{\text {by }} \pm 0.87$ & $6.22^{\mathbf{a y}} \pm 0.48$ & $6.89^{\mathrm{az}} \pm 0.24$ \\
\hline & & Cortisol & $2.87^{\mathrm{ay}} \pm 0.49$ & $4.72^{\mathbf{b v}} \pm 0.18$ & $10.92^{\mathrm{cy}} \pm 0.39$ & $11.54^{\mathrm{cz}} \pm 1.21$ & $8.08^{\text {du }} \pm 0.94$ \\
\hline & & T3 & $1.77^{\mathbf{a x}} \pm 0.06$ & $0.99^{\mathbf{b z}} \pm 0.11$ & $0.63^{\mathrm{cz}} \pm 0.10$ & $0.40^{\mathbf{d v}} \pm 0.07$ & $0.43^{\mathbf{d v}} \pm 0.02$ \\
\hline & & T4 & $32.05^{\mathrm{ay}} \pm 1.77$ & $34.21^{\mathrm{ay}} \pm 5.49$ & $21.43^{\mathrm{bz}} \pm 0.45$ & $32.14^{\mathbf{a v}} \pm 5.46$ & $32.39^{\mathrm{ay}} \pm 2.59$ \\
\hline & \multirow[t]{4}{*}{10} & GH & $6.68^{\mathrm{ay}} \pm 0.66$ & $5.99^{\mathrm{ay}} \pm 0.78$ & $4.89^{\text {by }} \pm 0.30$ & $7.80^{\mathrm{az}} \pm 0.98$ & $5.12^{\mathrm{ay}} \pm 0.32$ \\
\hline & & Cortisol & $3.95^{\mathrm{az}} \pm 0.98$ & $5.28^{\mathrm{bz}} \pm 0.39$ & $6.41^{\mathrm{cz}} \pm 0.65$ & $8.88^{\mathrm{dy}} \pm 0.56$ & $12.77^{\mathrm{ez}} \pm 0.63$ \\
\hline & & T3 & $1.31^{\mathrm{az}} \pm 0.05$ & $1.20^{\mathbf{a x}} \pm 0.07$ & $0.76^{\mathrm{bz}} \pm 0.13$ & $0.41^{\mathbf{c v}} \pm 0.17$ & $0.65^{\mathbf{b x}} \pm 0.09$ \\
\hline & & T4 & $35.49^{\mathrm{ay}} \pm 1.64$ & $36.50^{\mathrm{ay}} \pm 4.32$ & $35.99^{\mathbf{a x}} \pm 2.44$ & $29.97^{\mathbf{b z}} \pm 6.82$ & $25.86^{\mathbf{b x}} \pm 2.72$ \\
\hline & \multirow[t]{4}{*}{11} & GH & $6.6^{\mathrm{ay}} \pm 0.53$ & $6.95^{\mathrm{ay}} \pm 0.77$ & $4.97^{\text {by }} \pm 0.26$ & $6.58^{\mathbf{a y}} \pm 0.21$ & $6.55^{\mathrm{az}} \pm 0.72$ \\
\hline & & Cortisol & $4.13^{\mathrm{av}} \pm 0.44$ & $6.22^{b z} \pm 1.07$ & $13.22^{\mathrm{cx}} \pm 0.47$ & $7.99^{\mathrm{bx}} \pm 0.38$ & $19.40^{\mathrm{dw}} \pm 1.09$ \\
\hline & & T3 & $0.78^{\mathbf{a y}^{\prime}} \pm 0.02$ & $0.96^{\mathbf{b z}} \pm 0.01$ & $0.72^{\mathrm{az}} \pm 0.08$ & $1.09^{\mathrm{bz}} \pm 0.19$ & $0.95^{\mathbf{b x}} \pm 0.01$ \\
\hline & & T4 & $32.30^{\mathbf{a y}_{3.76}}$ & $29.13^{\mathbf{a z}} \pm 2.36$ & $27.69^{\mathbf{b y}} \pm 1.91$ & $35.63^{\mathrm{ay}} \pm 2.59$ & $18.41^{\mathrm{cz}} \pm 1.18$ \\
\hline
\end{tabular}

GH-Growth Hormone, T3-Triiodothyronine, T4-Thyroxine

$a$ and $b$ values with different superscript differ significantly $(\mathrm{P}<0.05)$ between columns $\mathrm{x}$ and $\mathrm{y}$ values with different superscript differ significantly $(\mathrm{P}<0.05)$ between rows 
Table.4 Mean \pm S.E.M values for body weight and dry matter intake ( $\mathrm{kg} / \mathrm{day})$ at different ages and seasons

\begin{tabular}{|c|c|c|c|c|c|c|c|}
\hline \multirow[t]{3}{*}{ Season } & \multirow{3}{*}{$\begin{array}{l}\text { Age } \\
\text { (mo } \\
\text { nths) }\end{array}$} & \multirow[t]{3}{*}{ Parameter } & \multicolumn{5}{|c|}{ Housing Conditions } \\
\hline & & & \multirow{2}{*}{$\begin{array}{l}\text { Control } \\
\text { Group }\end{array}$} & \multicolumn{4}{|l|}{ Field } \\
\hline & & & & Test Group I & $\begin{array}{l}\text { Test Group } \\
\text { II }\end{array}$ & $\begin{array}{l}\text { Test Group } \\
\text { III }\end{array}$ & $\begin{array}{l}\text { Test } \\
\text { Group IV }\end{array}$ \\
\hline \multirow[t]{4}{*}{ Spring } & \multirow[t]{2}{*}{6} & Body Weight & $78.85 \pm 5.56$ & $75.29 \pm 5.35$ & $70.35 \pm 1.67$ & $72.74 \pm 5.17$ & $68.79 \pm 1.45$ \\
\hline & & Feed intake & $1.81 \pm 0.13$ & $1.73 \pm 0.08$ & $1.61 \pm 0.10$ & $1.67 \pm 0.12$ & $1.57 \pm 0.13$ \\
\hline & \multirow[t]{2}{*}{7} & Body Weight & $85.02 \pm 5.59$ & $78.71 \pm 5.92$ & $73.14 \pm 1.91$ & $76.23 \pm 5.37$ & $71.58 \pm 1.73$ \\
\hline & & Feed intake & $1.96 \pm 0.13$ & $1.81 \pm 0.14$ & $1.68 \pm 0.74$ & $1.75 \pm 0.09$ & $1.64 \pm 0.04$ \\
\hline \multirow{4}{*}{$\begin{array}{l}\text { Dry } \\
\text { Hot }\end{array}$} & \multirow[t]{2}{*}{8} & Body Weight & $93.90 \pm 6.73$ & $89.92 \pm 6.68$ & $79.46 \pm 2.50$ & $84.92 \pm 6.46$ & $77.60 \pm 2.43$ \\
\hline & & Feed intake & $2.17 \pm 0.09$ & $2.08 \pm 0.16$ & $1.83 \pm 0.60$ & $1.96 \pm 0.16$ & $1.78 \pm 0.06$ \\
\hline & \multirow[t]{2}{*}{9} & Body Weight & $99.78 \pm 6.97$ & $93.96 \pm 6.75$ & $82.62 \pm 2.71$ & $88.59 \pm 6.17$ & $80.42 \pm 2.28$ \\
\hline & & Feed intake & $2.31 \pm 0.17$ & $2.17 \pm 0.16$ & $1.90 \pm 0.65$ & $2.05 \pm 0.08$ & $1.85 \pm 0.05$ \\
\hline \multirow{6}{*}{$\begin{array}{l}\text { Hot } \\
\text { Humid }\end{array}$} & \multirow[t]{2}{*}{10} & Body Weight & $108.34 \pm 7.20$ & $98.41 \pm 6.83$ & $85.51 \pm 2.62$ & $92.65 \pm 5.84$ & $83.64 \pm 2.17$ \\
\hline & & Feed intake & $2.52 \pm 0.17$ & $2.28 \pm 0.06$ & $1.97 \pm 0.63$ & $2.14 \pm 0.14$ & $1.93 \pm 0.05$ \\
\hline & \multirow[t]{2}{*}{11} & Body Weight & $117.14 \pm 6.17$ & $105.97 \pm 6.13$ & $88.65 \pm 2.23$ & $98.64 \pm 6.28$ & $87.11 \pm 2.03$ \\
\hline & & Feed intake & $2.73 \pm 0.08$ & $2.46 \pm 0.15$ & $2.05 \pm 0.54$ & $2.29 \pm 0.07$ & $2.01 \pm 0.15$ \\
\hline & \multirow[t]{2}{*}{12} & Body Weight & $128.73 \pm 4.48$ & $112.97 \pm 5.26$ & $92.42 \pm 2.12$ & $104.12 \pm 7.30$ & $89.37 \pm 1.57$ \\
\hline & & Feed intake & $3.01 \pm 0.11$ & $2.63 \pm 0.13$ & $2.14 \pm 0.51$ & $2.42 \pm 0.08$ & $2.06 \pm 0.14$ \\
\hline
\end{tabular}

$a$ and $b$ values with different superscript differ significantly $(\mathrm{P}<0.05)$ between columns

$\mathrm{x}$ and $\mathrm{y}$ values with different superscript differ significantly $(\mathrm{P}<0.05)$ between rows

\section{Endocrine profile (ng/ml)}

The pattern of GH secretion was irregular and the peak values were obtained at the age of 9 months Test Group I, at the age of 8 months under Test Group III, at the age of 11 months under Test Group II and at the age of 10 months under Test Group IV (Table 3). Vicari et al., (2008) reported a similar irregular pattern of $\mathrm{GH}$ secretion in growing calves in which basal and peak GH concentrations were unrelated to either the feed frequency or metabolizable energy values. All the groups exhibited a different pattern for plasma cortisol activity. In control group, highest levels of cortisol was observed during $9^{\text {th }}$ month of age during hot humid season, whereas under field conditions the maximum values for plasma cortisol were reported under tree during hot humid season followed by test group 2 and 3 . The overall range for plasma cortisol was lowest under Test Group I. The higher levels of plasma cortisol were observed particularly during hot dry and hot humid seasons. Significant $(\mathrm{P}<0.01)$ differences in cortisol levels were observed between different housing systems. The present findings are similar to those reported by Christison and Johnson (1972), Habeeb et al., (1992, 2001), Muller et al., (1994), ZiaUr-Rahman et al., (1997), and Dhami et al., (2006). The plasma $T_{3}$ and $T_{4}$ in different housing groups. The lowest $\mathrm{T}_{3}$ values were observed during hot humid and hot dry season with higher variability under all the field housing groups as compared to conventional housing system. The higher values for plasma $\mathrm{T}_{3}$ were observed particularly during hot dry and hot humid seasons with peak values during hot humid season under all housing systems as compared to spring season. Significant $(\mathrm{P}<0.01)$ differences in plasma $\mathrm{T}_{3}$ 
were observed between different housing systems. Under field conditions the minimum plasma $\mathrm{T}_{4}$ levels were reported among animals housed under tree during hot humid season followed by test group 2 and 3 during hot dry season. Significant $(\mathrm{P}<0.01)$ differences in $\mathrm{T}_{4}$ levels were observed between different housing systems. In present study it was observed that season, housing type and temperature can alter thyroid activity under heat stress as suggested by Collier et al., (1982) and Gomila et al., (1977). Yousef and Johnson (1966) also reported that dairy cows exposed to chronic high environmental temperatures had depressed thyroid activity.

\section{Body weight (Kg) and feed intake (Kg/day)}

The BW and FI was found to be significantly $(\mathrm{P}<0.01)$ affected by the housing condition provided to each study group (Table 4). The highest of the average daily weight gain was recorded control group whereas the Test group IV exhibited the poorest growth rates. When compared among all the test groups from villge, the Test Group I was found to be excellent as far as feed intake and body wight gain was concerned. Increased in the DMI with the advancement of age was recorded increased under all the housing groups. The feed intake in the present study seems to be effected by different seasons, age groups and housing systems. Ragsdale et al., (1948) and Mullick (1964) has also reported reduction in voluntary feed intake of animals under stress conditions as compared to thermo neutral temperatures.

\section{References}

Andrighetto I., Gottardo F., Andreoli D. and Cozzi G. 1999. Effect of type of housing on veal calf growth performance, behaviour and meat quality. Livestock Prod. Sci. 57:137145.
Blackshaw J. K. and Blackshaw A.W. 1994. Heat stress in cattle and effect of shade on the production and behavior, A review. Aust. J. Exp. Agri. 34: 285-295.

Broucek J., Kisac P. and Uhrincat M. 2009. Effect of hot temperatures on the hematological parameters, health and performance of calves. Int $J$ Biometeorol. 53:201-208.

Christison G.I. and Johnson H.D. 1972. Cortisol turnover in heat stressed cows. J. Anim. Sci. 53: 1005-1010.

Chua B., Coenen E., Delen J. van and Weary D. M. 2002. Competition for teats and feeding behaivor by group-housed dairy calves. J. Dairy Sci. 85: 360-364.

Coban O. and Sabuncuoglu N. 2005. Blood charecteristics of dairy calves as affected by age, breed and types of barn. J. of Anim. and Vet. Adv. 4 (4): 459-461.

Coleman D. A., Moss B. R. and McCaskey T. A. 1996. Supplemental shade for dairy calves reared in commercial calf hutches in a southern climate. $J$. of dairy sci. 79(11): 2038-2043.

Collier R. J., Breede D. K., Thatcher W. W., Israel L. A. and Wilcox C. J. 1982. Influence of environment and its modification on dairy animal health and production. J. Dairy Sci. 65: 22132227.

Dandage S. D. 2009. Estimates of thermal load and heat exchange in cattle and buffaloes (Unpublished master's thesis). National Dairy Research Institute (Deemed University), Karnal, India.

Dhami A. J., Parmar B. C., Raval R., Gupta R. S. and Trivedi M. M. 2006. Effect of challenge feeding during summer on the productive and reproductive performance and blood biochemical, metabolic and hormonal profile of crossbred lactating cows. Int. J. Cow Sci. 2(2): 0973-2241. 
Duguma, B., Kechero, Y. and Janssens, G.P. J. (2012) Survey of Major Diseases Affecting Dairy Cattle in Jimma Town, Oromia, Ethiopia. Global Veterinaria, 8(1): 62-66.

Gomila L. F., Roussel J. D. and Beatty, J. F. 1977. Effect of zone cooling on milk yield, thyroid activity and stress indicators. J. Dairy Sci. 60: 129-132

Guyton A. C. 1986. Textbook of Medical Physiology. (7th Ed) W. B. Saunders Company, Philadelphia. pp. 849-851.

Habeeb A. A. M., Aboulnaga A. J., Kamal T. H. 2001. Heat-induced changes in body water concentration, $\mathrm{T}_{3}$, cortisol, glucose and cholesterol levels and their relationships with thermo neutral bodyweight gain in Friesian calves. Proceedings of 2nd International Conference on Animal Production and Health in Semi-arid Areas, pp. 97-108. El-Arish, North Sinai, Egypt.

Habeeb A. A. M., Marai I. F. M., Kamal T. H. 1992. Heat stress. In: Phillips, C., Pigginns, D. (Eds.), Farm Animals and the Environment. CAB International, Wallingford, UK, pp. 27-47.

Heinrichs A. J., Heinrichs B. S and Harel O. 2005. A prospective study of calf factors affecting age, body size, and body condition score at first calving of Holstein dairy heifers. J. Dairy Sci. 88:2828-2835.

Hill T. M., Bateman II H. G., Aldrich J. M. and Schlotterbeck L. 2007.Effects of milk replacers and bedding materials for calves in a cold and naturally ventilated nursery. Professional Anim. Scientist. 23: 656-664.

Ingole S. D., Deshmukh B. T., Nagvekar A. S. and Bharucha, S. V. 2012. Serum Profile of Thyroid Hormones from Birth to Puberty in Buffalo Calves and Heifers. J. of Buff. Sci. 1: 39-49.
Kurtz E. 1961. Raising dairy herd replacements. South Dakota State Coll. Coop. Ext. Serv. F.S., 67.

Marai I. F. M., El-Masry K. A. and Nasr A. S. 1995. Heat stress and its amelioration with nutritional, buffering, hormonal and physiological techniques for New Zealand White rabbits maintained under hot summer conditions of Egypt. Options Mediterr. 8: 475-487.

Marai I. F. M., Habeeb A. A. M., Daader A. H. and Yousef H. M. 1997. Effects of diet supplementation and body cooling on Friesian calves reared in high ambient temperature in the Eastern Desert of Egypt. J. Trop. Anim. Health Prod. 4: 201-208.

Muller C. J. C., Botha J. A., Coetzer W. A. and Smith W. W. 1994. Effect of shade on various parameters of Friesian cows in a Mediterranean climate in South Africa. 2. Physiological responses. South Afric. J. Anim. Sci. 24: 56-60.

Mullick D. N. 1964. A study on the metabolism of food nutrients in cattle and buffaloes under climatic stress. Arid Zone Res. 14:137.

Nardone A., Ronchi B., Lacetera N. and Bernabucci U. 2006. Climatic effects on productive traits in Livestock. Veterinary Research Communications 30 (Suppl 1): 75-81.

O’Brien M. D., Rhoads R. P., Sanders S. R., Duff G. C. And Baumgard, L. H. 2010. Metabolic adaptations to heat stress in growing cattle. Dome. Anim. Endo. 38: 86-94.

Pereira B. M. J., Balsubramanian K. and Govindarajulu P. 1983. Influence of hypothyroidism on epididymal enzymes involved in carbohydrate metabolism. Studies in pubertal and adult rats. J. Androl. 6: 294-304.

Prasanpanich S., Semicha S., Tunsaringkarn K., Thwaites C. J. and Vajnabukka C. 2002. Physiological responses of 
lactating cows under grazing and indoor feeding conditions in the tropics. $J$. Agric. Sci. 138: 341-344.

Ragsdale A. C., Brody S., Thompson H. L. and Worstell D. M. 1948. Environmental physiology with special reference to domestic animals. II. Influence of temperature, 500 to 1050 F., on milk production and feed consumption in dairy cattle. Mo. Agr. Exp. Sta. Res. Bul. 425.

Razzaque M. A., Abbas S., Al-Mutawa T. and Bedair M. 2009. Performance of preweaned female calves confined in housing and open environment hutches in Kuwait. Pakistan Vet. J. 29(1): 1-4.

Shebaita M. K., Kamal T. H. 1973. In vivo body composition in ruminants. I. Blood volume in Friesian and water buffaloes. Alex J Agric Res 1:329-350

Silanikove N. 1987. Impact of shade in hot Mediterranean summer on feed intake, feed utilization and body fluid distribution in sheep. Appetite 9: 207 215.

Singh K. 1983. Effect of heat stress on some blood constituents in cross bred heifers Ind J Anim Sci. 53:355.
Spain J. N. and Spiers D. E. 1996. Effects of supplemental shade on thermoregulatory response of calves to heat challenge in a hutch environment. Journal of dairy science. 79(4): 639646.

Vicari T., van den Borne J.J.G.C., Gerrits W. J. J., Zbinden Y. \& Blum J. W. 2008 Separation of protein and lactose intake over meals dissociates postprandial glucose and insulin concentrations and reduces postprandial insulin responses in heavy veal calves. Domestic Animal Endocrinology 34 182-195.

Yousef, M.K. and Johnson, H.D. 1966. Blood thyroxine degradation rate in cattle as influenced by temperature and feed intake. Life Sci. 5: 1349.

Zia-Ur-Rahman, I. U., Haq, I., Javed, Mushtaq-Ul-Hassan, Z. H., Naqvi, M M. and Jaivi, A. M. 1997. Hormonal and haematological profiles in buffalo after transport, handling and slaughter stress. In: Proc. Fifth World Buffalo Congress. Caserta, Italia, 961-965

\section{How to cite this article:}

Vaibhav N. Sanap, Ashutosh Ludri, Nazir Ahmad Mir, Bharath Kumar and Kamlesh Kumar Mittal. 2018. Physiological Performance of Crossbred Cattle Calves (Karan Fries) under Different Housing Conditions during Different Seasons. Int.J.Curr.Microbiol.App.Sci. 7(11): 2738-2748. doi: https://doi.org/10.20546/ijcmas.2018.711.314 